\title{
The Prevalence of Ketogenic Diet among Adults with Chronic Disease in Saudi Arabia
}

\author{
Khalid Abdullah Algamdi ${ }^{1}$, Sajidah Hisham Abu Alsaud ${ }^{* 2}$, Ghada Badr Alghubaini ${ }^{2}$, Muna Mustafa Alsaggaf ${ }^{2}$, \\ Ibtisam Ahmed Altalhi ${ }^{3}$, Jood Sami Asali ${ }^{3}$, Shada Mohammed Abyad ${ }^{4}$, Khames T. Alzahrani ${ }^{5}$ \\ ${ }^{1}$ Specialized Polyclinic, Ministry of Interior Security Forces Medical Services, Jeddah, Kingdom of Saudi Arabia \\ ${ }^{2}$ Medical Intern, Ibn Sina National Collage, Jeddah, Saudi Arabia \\ ${ }^{3}$ Medical Student, Taif University, Taif, Saudi Arabia \\ ${ }^{4}$ Medical Student Umm Al-Quraa University, Mecca, Saudi Arabia \\ ${ }^{5}$ Restorative Department, BDS, PGD Endo, Ministry of Health, Saudi Arabia
}

*Corresponding author Sajidah Hisham Abu Alsaud; Sajida.abAbstractualsaud@gmail.com

Received 31 December 2020;

Accepted 20 January 2021;

Published 21 January 2021

\begin{abstract}
Introduction: Ketogenic diet was first approached diet in treatment of epilepsy in children, later on, many studies showed its positive effect on adults who have chronic diseases and obesity. The main aim of the diet is to allow the body to reach the state of ketosis by generating energy from fat rather than glucose, and this happen by lowering the carbohydrates, adding moderate protein and high fat, thus letting the body burn fat for fuel. Since more than 50 years studies linked consumption of saturated fat with cardio-metabolic disease risk and obesity, and nowadays many studies have shown a positive impact of consumption of saturated fat. Therefore, this is a study to determine the prevalence of Saudi adult with chronic disease who follow a ketogenic diet. Methods: This was an observational cross-sectional study based on web-survey questionnaires developed by developed originally by author with high confidence level. The sample size was 479 estimated using the Qualtrics calculator with a confidence level of $95 \%$ and a margin of error of $5 \%$. The questionnaire is divided into four categories. We started the questioner with demographic factors including age, nationality, dental degree date and in which country, work setting and cariology course attendance. 2nd it was about 3 clinical scenarios. Data were collected using Google forms and prepared for analysis using Microsoft Excel. Statistical analysis was performed using the SPSS software. Results: The total number of the current study participants was 464, the highest percentage (33.90\%) were in the age group of $40-50$ years old, $44.80 \%$ were in the weight range of $61-80 \mathrm{~kg}$, while $11.90 \%$ have a weight of $>100 \mathrm{~kg}$, and half (50\%) of the participants have a height range of $151-160 \mathrm{~cm}$. Most $(71 \%)$ of the participants have a college educational level, and $44.30 \%$ were employees. The prevalence of hypercholesterolemia and diabetes among the respondents was $23.70 \%$, and $2.50 \%$, respectively. The overall prevalence of metabolic syndrome was $2.30 \%$. Almost half (50.10\%) of the participants reported having obesity, and $19.10 \%$ were hypertensive. Conclusion: Ketogenic diet in this review showed a positive results within the utilization in chronic illnesses and obesity. In any case, The longterm hazard related with adverse impacts is unknown; therefore, advance studies may be valuable. Future randomized clinical trials are required to affirm the viability of the ketogenic diet in different sorts of adults' chronic illness and to supply advance data on a few uncertain commonsense issues, i.e., how long the ketogenic diet ought to be proceeded.
\end{abstract}

\section{Keywords: Ketogenic Diet, Saudi Arabia}

\section{Introduction}

The ketogenic diet $(\mathrm{KD})$ is defined as a diet high in fat, appropriate in protein, and low in carbohydrates ${ }^{[1]}$. Original research into a ketogenic diet started within the early 1900 s as a way to manage epilepsy and minimize seizure movement and over the final few decades has given prove for the helpful potential of ketogenic diets in numerous pathological conditions, including diabetes, PCOS, acne, neurologic illnesses (epilepsy, Alzheimer's, CVA), cancer, and the improvement of respiratory and cardiovascular illness risk factors ${ }^{[2]}$.
As appeared in Table 1, glycaemia indeed in spite of the fact that decreased remains inside physiological levels ${ }^{[3]}$.

Table 1: Blood levels during a normal diet, ketogenic diet and diabetic ketoacidosis ${ }^{[3]}$.

\begin{tabular}{|l|l|l|l|}
\hline Blood Levels & $\begin{array}{l}\text { Normal } \\
\text { Diet }\end{array}$ & $\begin{array}{l}\text { Ketogenic } \\
\text { Diet }\end{array}$ & $\begin{array}{l}\text { Diabetics } \\
\text { Ketoacidosis }\end{array}$ \\
\hline Glucose $(\mathrm{mg} / \mathrm{dl})$ & $80-120$ & $65-80$ & $>300$ \\
Insulin $(\mu \mathrm{U} / \mathrm{L})$ & $6-23$ & $6.6-9.4$ & $\approx 0$ \\
KB conc $(\mathrm{mmol} / \mathrm{L})$ & 0.1 & $7 / 8$ & $>25$ \\
$\mathrm{pH}$ & 7.4 & 7.4 & $<7.3$ \\
\hline
\end{tabular}


Above all, a study in 2019, show that patients who need a fast wight reduction over a short period, such as individuals with mild to extremem obesity and cardiovascular risk factor, the VLCKD is recommended as a quite effective therapeutic tool; Also VLCKD has ability in remission of T2D particularly in obese patients with brief sickness period ${ }^{[4]}$. Also in a systematic review with metaanalysis, Bezerra Bueno et al. looked into 13 randomized controlled trials (RCT), which included add up to of 1569 subjects and compared VLCKD $(<50 \mathrm{~g}$ carb/day) with low fat diet (LFD, $<30 \% \mathrm{kcal}$ from fat) in overweight and obese adult. The included RCTs had a least follow-up of 12 months. The primary result for their meta-analysis was body weight; auxiliary results included lipid profile (triglycerides, HDL, LDL), systolic and diastolic blood weight, fasting blood sugar, insulin, HbA1c, and CRP. Analysis revealed that VLCKD accomplished greater long-term decreases in body weight, triglycerides, and diastolic blood pressure, and more prominent increases in HDL and LDL cholesterol compared to a low-fat diet ${ }^{[2]}$.

Moreover, a study reported that the observed risk proportion for the association between saturated fat and CVD occasions does not fit a connection with plasma LDL-cholesterol, but or maybe, is related to the proportion of apolipoprotein $\mathrm{B}$ (ApoB) to ApoA1, which may be a degree related to atherogenic molecule concentration (ApoB is found in LDL and exceptionally low- density lipoprotein particles, and ApoA1 is found in HDL particles); in reality, this proportion is lower in those with higher saturated fat admissions ${ }^{[5]}$. Another study in 2016, show that highglycemic car-bohydrates or high overall proportions of carbohydrates within the diet are the key biological relates of CVD risks. These findings strikingly contradict the conventional 'saturated fat hypothesis' but in reality, they are consistent with the prove gathered from observational studies about that points to both high glycemic index and high glycemic load as vital triggers of CVDs ${ }^{[6]}$. In 2015, a clinical trail study among 148 obese adults deliver through 12 months, use of low carbohydrates diet result in improve inflammatory marker and cardiovascular disease marker ${ }^{[7]}$. Also, a Study have been published on Effects of a ketogenic diet in overweight women with polycystic ovary syndrome and showed that the ketogenic diet can be used as therapy in PCOS ${ }^{[3]}$.

\section{Materials and Methods}

This is an observational cross-sectional study conducted in Saudi Arabia to identify the prevalence of ketogenic diet among Saudi adults with chronic disease.

The sample size was 464, consisted of Saudi adults with chronic disease with 95\% confidence level and 5\% marginal errer. The Inclusion criteria of this study 18 years and older with Chronic diseases: diabetes mellitus, obesity, dyslipidemia, cardiacdiseases, insulin resistance, poly cystic ovaries syndrome.Agree to Participate.

The survey was a self-administered questionnaire in Arabic added online on google forum, containing questions regarding keto diet among Saudi population with chronic disease, and it include age, gender, occupation, education level, type of diet, and chronic diseases like diabetes mellitus, hypertension, obesity, cardiovascular disease, dyslipidemia, polycystic ovaries syndrome, insulin resistance. The data collected by computer using the "Microsoft Office Excel Software" program (2019) for windows. Will then be transferred to the Statistical Package of Social Science Software (SPSS) program, version 20 (IBM SPSS Statistics for Windows, Version 20.0. Armonk, NY: IBM Corp). This research was approved by the institutional review board by The Directorate of Heath Affairs In Saudi Arabia.

\section{Results}

The total number of the current study participants was 464 , the highest percentage (33.90\%) were in the age group of 40-50 years old, $44.80 \%$ were in the weight range of $61-80 \mathrm{~kg}$, while $11.90 \%$ have a weight of $>100 \mathrm{~kg}$, and half $(50 \%)$ of the participants have a height range of $151-160 \mathrm{~cm}$. Most $(71 \%)$ of the participants have a college educational level, and $44.30 \%$ were employees. The prevalence of hypercholesterolemia and diabetes among the respondents was $23.70 \%$, and $2.50 \%$, respectively. The overall prevalence of metabolic syndrome was $2.30 \%$. Almost half $(50.10 \%)$ of the participants reported having obesity, and $19.10 \%$ were hypertensive.

Almost one-third $(32.40 \%)$ of the participants reported following the ketogenic diet, and only $5 \%$ of them reported following it under medical supervision. For duration, $13.60 \%$ reported being on the ketogenic diet for less than a month, and $13.40 \%$ for less than 6 months, while $20 \%$ reported improvement in their medical condition after months of following the ketogenic diet. The largest proportion of the participants did not follow any diet before at $48.50 \%$, while $25.50 \%$ reported benefiting from certain diet that led them quitting the medications they were using. Serious diet side effects were reported by $8.20 \%$ of the respondents and $65.80 \%$ think that saturated fats are wrongly shown for their negative health impact. Almost 30.90\% reported noticing physical and health benefits after trying the ketogenic diet.

There was a statistically significant (P 0.012) difference in the prevalence of following ketogenic diet by age group, where it was the highest at $35.10 \%$ among those aged 29-39 years old. Besides, a statistically significant $(\mathrm{P}<0.001)$ higher percentage (38.90\%) of those aged 40-50 years old think that different sources of saturated and unsaturated fats affect their health differently.

A borderline significant difference by knowledge level was noticed in relation to the use of ketogenic diet (P 0.055), since the majority $(79.40 \%)$ of those who followed ketogenic diet have a college educational level, while none of the non-educated ones followed it. Similarly, a significantly higher percentage of college participants reported gaining health benefits after following a certain diet that lead them quitting medications they were using, and think that different sources of saturated and unsaturated fats affect their health differently at $68 \%$, and $67.90 \%$ respectively, and $P$ values $<0.05$.

Following ketogenic diet was significantly correlated with participants' occupation, being highly prevalent among employees at $52.70 \%$ compared to $32.10 \%$ for non-employees and $15.30 \%$ among students, with a P-value of 0.021 . In addition, significant differences were found between the different occupations by the duration of the ketogenic diet and time spent on diet till improvement. The highest rate of experiencing side effects from diet was reported by unemployed at $45.50 \%$ followed by students at $30.30 \%$, while employed subjects showed the lowest prevalence of side effects due to diet at $24.20 \%$, with a P-value of 0.019 .

The results of the current study revealed that following the ketogenic diet was significantly correlated with obesity and chronic diseases. A significantly (0.047) higher percentage of obese and chronic diseases participants reported following a ketogenic diet at $70.20 \%$. In addition, the duration of being on a ketogenic diet till improvement was significantly higher among obese subjects and those with chronic disease. Around $70 \%$ of obese and chronic disease participants reported noticing the physical and health 
benefits after trying the ketogenic diet compared to $29.60 \%$ of the other group with a significant $\mathrm{P}$ value at 0.039 .

\section{Discussion}

The current study estimated the prevalence of ketogenic diet among adults in Saudi Arabia, and it was found that almost onethird of the current study participants are following it at $32.40 \%$.

Recently, the Ketogenic diet has shown to have benefits besides being used for the treatment of epilepsy. Such benefits include weight loss and reversal/control of type 2 diabetes ${ }^{[8]}$.

Besides a positive effect on weight loss, studies have shown that low-carbohydrate ketogenic diets also reduce serum triglycerides dramatically ${ }^{[9]}$. Such reported benefits were in line with our results, where a weight loss and health benefits including quitting certain medications were reported. However, even though the use of ketogenic diets in weight management has gained tremendous popularity and achieved success, it has also generated several arguments ${ }^{[9]}$. Ketogenic diet side effects include muscle cramps, bad breath, changes in bowel habits, keto-flu, and loss of energy ${ }^{[10]}$. Henceforth, it is essential to monitor individuals on keto-diet closely once or twice a month for blood glucose, ketones cardiac and other parameters is essential ${ }^{[9]}$. Despite these recommendations, most of the participants in the current study are not following diet under medical supervision.

A previously published study addressed the long-term effect of the ketogenic diet showed it significantly reduced the body weight and body mass index. Also, it decreased the level of triglycerides, LDL cholesterol, and blood glucose, and increased the level of HDL cholesterol ${ }^{[11]}$. Our finding might indirectly augment these results, as we did not specify the change in lipid profile per se, but the participants reported a decrease in chronic disease symptoms. For side effects, ${ }^{[11]}$ reported that administering a ketogenic diet for a relatively long period did not produce any significant side effects. Therefore, they confirmed the safety of the ketogenic diet for a longer period than previously demonstrated. Similarly, the laboratory results from another study showed that the ketogenic diet modified the risk factors for heart disease in obese patients ${ }^{[12]}$.

There is an argument against the consumption of a high-fat diet, which is that it causes obesity. The main concern is whether a high percentage of dietary fat promotes weight gain more than a low percentage of fat intake. Since fat has a higher caloric density than carbohydrates, it is thought that the consumption of a high-fat diet will be accompanied by a higher energy intake ${ }^{[13]}$. In contrast, many other laboratories have observed that a ketogenic diet can be used as a therapy for weight reduction in obese patients ${ }^{[14]}$. In the current study, around two-thirds of the participants think saturated fats are wrongly shown for their negative health impact.

In a study among diabetic obese subjects, the authors reported that ketogenic diet has beneficial effects in obese diabetic subjects following its long-term administration ${ }^{[15]}$. Fortunately, following a ketogenic diet may improve many features of metabolic syndrome including better cholesterol values, as well as reduced blood sugar and blood pressure ${ }^{[16]}$. In a 16-week study, 17 of 21 people on a ketogenic diet were able to discontinue or decrease diabetes medication dosage. Study participants also lost an average of $8.7 \mathrm{~kg}$ and reduced their waist size, triglycerides, and blood pressure ${ }^{[17]}$.

\section{Conclusion}

Ketogenic diet in this review showed a positive result within the utilization in chronic illnesses and obesity. In any case, the longterm hazard related with adverse impacts is unknown; therefore, advance studies may be valuable. Future randomized clinical trials are required to affirm the viability of the ketogenic diet in different sorts of adults' chronic illness and to supply advance data on a few uncertain common-sense issues, i.e., how long the ketogenic diet ought to be proceeded.

\section{Conflicts of Interest}

The authors have no conflicts of interest to declare.

\section{Source of Funding}

This study did not receive any specific grant from funding agencies in the public, commercial, or not-for-profit sectors.

\section{Ethical approval}

Directorate of Heath Affairs in Saudi Arabia

\section{References}

[1] Gupta L, Khandelwal D, Kalra S, Gupta P, Dutta D, Aggarwal S. Ketogenic diet in endocrine disorders: Current perspectives. Vol. 63, Journal of Postgraduate Medicine. Medknow Publications; 2017. p. 242-51.

[2] Gershuni VM, Yan SL, Medici V. Nutritional Ketosis for Weight Management and Reversal of Metabolic Syndrome. Vol. 7, Current Nutrition Reports. Current Science Inc.; 2018. p. 97-106.

[3] Paoli A, Mancin L, Giacona MC, Bianco A, Caprio M. Effects of a ketogenic diet in overweight women with polycystic ovary syndrome. J Transl Med [Internet]. 2020;18(1):1-11. Available from: https://doi.org/10.1186/s12967-020-02277-0

[4] Caprio M, Infante M, Moriconi E, Armani A, Fabbri A, Mantovani G, et al. Very-low-calorie ketogenic diet (VLCKD) in the management of metabolic diseases: systematic review and consensus statement from the Italian Society of Endocrinology (SIE). J Endocrinol Invest. 2019;42(11):1365-86.

[5] Astrup A, Magkos F, Bier DM, Brenna JT, de Oliveira Otto MC, Hill JO, et al. Saturated Fats and Health: A Reassessment and Proposal for Food-based Recommendations: JACC State-of -the-Art Review. J Am Coll Cardiol [Internet]. 2020 Jun; Available from: https://linkinghub.elsevier.com/retrieve/pii/S0735109720 356874

[6] Grasgruber P, Sebera M, Hrazdira E, Hrebickova S, Cacek J. Food consumption and the actual statistics of cardiovascular diseases: An epidemiological comparison of 42 European countries. Food Nutr Res. 2016 Sep $27 ; 60$.

[7] Hu T, Bazzano LA. The low-carbohydrate diet and cardiovascular risk factors: Evidence from epidemiologic studies. Vol. 24, Nutrition, Metabolism and Cardiovascular Diseases. Elsevier; 2014. p. 337-43.

[8] McKenzie AL, Hallberg SJ, Creighton BC, Volk BM, Link TM, Abner MK, et al. A Novel Intervention Including Individualized Nutritional Recommendations Reduces Hemoglobin A1c Level, Medication Use, and 
Weight in Type 2 Diabetes. JMIR Diabetes. 2017;2(1):e5.

[9] Shilpa J, Mohan V. Ketogenic diets: Boon or bane? Vol. 148, Indian Journal of Medical Research. Wolters Kluwer Medknow Publications; 2018. p. 251-3.

[10] Barañano KW, Hartman AL. The ketogenic diet: Uses in epilepsy and other neurologic illnesses. Vol. 10, Current Treatment Options in Neurology. 2008.

[11] Dashti HM, Mathew TC, Hussein T, Asfar SK, Behbahani A, Khoursheed MA, et al. Long-term effects of a ketogenic diet in obese patients. Exp Clin Cardiol. 2004;9(3).

[12] Dashti HM, Bo-Abbas YY, Asfar SK, Mathew TC, Hussein T, Behbahani A, et al. Ketogenic diet modifies the risk factors of heart disease in obese patients. Nutrition. 2003;19(10).
[13] Prentice AM. Manipulation of dietary fat and energy density and subsequent effects on substrate flux and food intake. Am J Clin Nutr. 1998;67(3 SUPPL.):535-41.

[14] Westman EC, Mavropoulos J, Yancy WS, Volek JS. A review of low-carbohydrate ketogenic diets. Vol. 5, Current Atherosclerosis Reports. 2003.

[15] Dashti HM, Mathew TC, Khadada M, Al-Mousawi M, Talib H, Asfar SK, et al. Beneficial effects of ketogenic diet in obese diabetic subjects. Mol Cell Biochem. 2007 Aug;302(1-2):249-56.

[16] Volek JS, Phinney SD, Forsythe CE, Quann EE, Wood RJ, Puglisi MJ, et al. Carbohydrate restriction has a more favorable impact on the metabolic syndrome than a low fat diet. Lipids. 2009;44(4):297-309.

[17] Yancy WS, Foy M, Chalecki AM, Vernon MC, Westman EC. A low-carbohydrate, ketogenic diet to treat type 2 diabetes. Nutr Metab. 2005;2. 